\title{
Lautäußerung und Verhalten des Azoren-Buchfinken (Fringilla coelebs moreletti Pucheran)
}

\author{
von Sigrid Knecht und UlRich Scheer
}

Mit 16 Abbildungen

Eingegangen am 16. 6. 1967

In h a $1 \mathrm{t}$ : Einleitung und Methode S. 155. - Brutbiologie S. 155. - Motivgesang S. 157. Sozialruf (Social Call) S. 161. - Entwicklung des Sozialrufs S. 164. - Brutstimmungsruf (Regenruf) S. 165. - Flugruf S. 166. - Alarmruf eines Jungvogels S. 167. - Bestimmung der Reviergröße S. 167. - Zusammenfassung S. 168. - Summary S. 168. - Literaturverzeichnis S. 169.

\section{Einleitung und Methode}

Über die Lautäußerung des Azoren-Buchfinken gibt es bisher nur wenige Untersuchungen (Marler und Boatman 1951, Marler 1952); kurze Notizen finden sich bei MARLer (1956 a), Thorpe (1958) und Knecht (1961). MARLER und BOATMAN schien der auf Pico gehörte Gesang einfacher als der kontinentaler Buchfinken, auch waren manche Rufe stark abweichend. KNECHT fragte, ob es für die Azoren typische Gesangsvarianten und spezielle Rufe gibt, so daß man von „Rassengesang" und „Rassenrufen" sprechen könnte.

Während unseres Aufenthalts auf den Azoren vom 29. 4. - 28. 8. 1964 nahmen wir möglichst viele Gesänge und Rufe der dortigen Buchfinkenrasse aufs Band, registrierten jedes Nest, das wir fanden, seine Lage, Maße und Art des Genists, sowie Anzahl, Größe und Farbe der Eier.

Wir verwendeten das batteriebetriebene Stellavox-Tonbandgerät mit Vollspur, $19 \mathrm{~cm} / \mathrm{sec}$, und zur Wiedergabe einen großen Lautsprecher. Im Brennpunkt eines Parabolspiegels von $80 \mathrm{~cm} \varnothing \mathrm{sa}$ das dynamische Mikrofon mit einem Frequenzgang von 40-15 000 Hz. Durch eine Visiereinrichtung peilten wir den Vogel an; wenn er nicht zu sehen war, richteten wir uns nach dem Lautstärkeanstieg in den Kopfhörern. Noch auf $200 \mathrm{~m}$ Entfernung erhielten wir einwandfreie Tonaufnahmen (vgl. Wahlströм 1960, Ausoвsкy 1964).

Für das Interesse und die Unterstützung bei unserer Arbeit danken wir der Fundação Calouste Gulbenkian (Lissabon), der Deutschen Forschungsgemeinschaft sowie verschiedenen Behörden und Privatpersonen auf den Azoren. Zur Aufnahme der Klangspektrogramme diente das Herrn Prof. Dr. O. KoeHLer von der Deutschen Forschungsgemeinschaft zur Verfügung gestellte Gerät.

\section{Brutbiologie}

Die Buchfinkennester sind meist sehr sorgfältig aus Würzelchen, Gräsern, Moos, Flechten, Ericazweigen und Kiefernnadeln, manchmal auch Stückchen von Eucalyptus- oder Cryptomerenrinde (Cryptomeria japonica) gebaut. Einige Nester enthalten außen nur Moos und Flechten, andere nur Wurzeln und Gräser. Ausgepolstert sind sie regelmäßig mit Rinderhaaren, Federn und Gräsern, bisweilen mit Flughaaren von Kompositensamen.

Bei 54 Nestern war die Mulde 4-7, im Mittel 5,7 cm weit und 3-5, im Mittel 4,3 cm tief. Die Nesthöhe betrug 6-13, im Mittel 8,1 cm, der $\varnothing 6-12$, im Mittel $9,2 \mathrm{~cm}$. Sie standen meist 2-6, selten $10 \mathrm{~m}$ hoch in Astgabeln oder wirteligen Verzweigungen von Bäumen und Büschen, eines ruhte unmittelbar auf einem Kiefernast. An geeigneten Stellen lagen die Nester sehr eng nebeneinander, drei im Abstand von je $20 \mathrm{~m}$, bei Sete Cidades (S. Miguel) fünf auf einer Wegstrecke von $144 \mathrm{~m}$. 
Von 43 in den Monaten Mai bis Juli kontrollierten Nestern enthielten 23 drei, 11 zwei Eier oder Junge, 7 ein Ei oder Junges, 2 vier Eier. Die Eier sind grünlichweiß bis beige, mit rotbraunen Flecken und Schlieren, im Mittel 20,5 mm $\times 15,0 \mathrm{~mm}$ groß (wie die deutscher Buchfinken, Niethammer 1937).

Am 26. 5. sahen wir Junge ausfliegen, die noch einige Zeit außerhalb des Nests von beiden Eltern gefüttert wurden. Am 18. 7. fanden wir noch Nester mit Jungen; es gibt wohl zwei Bruten.
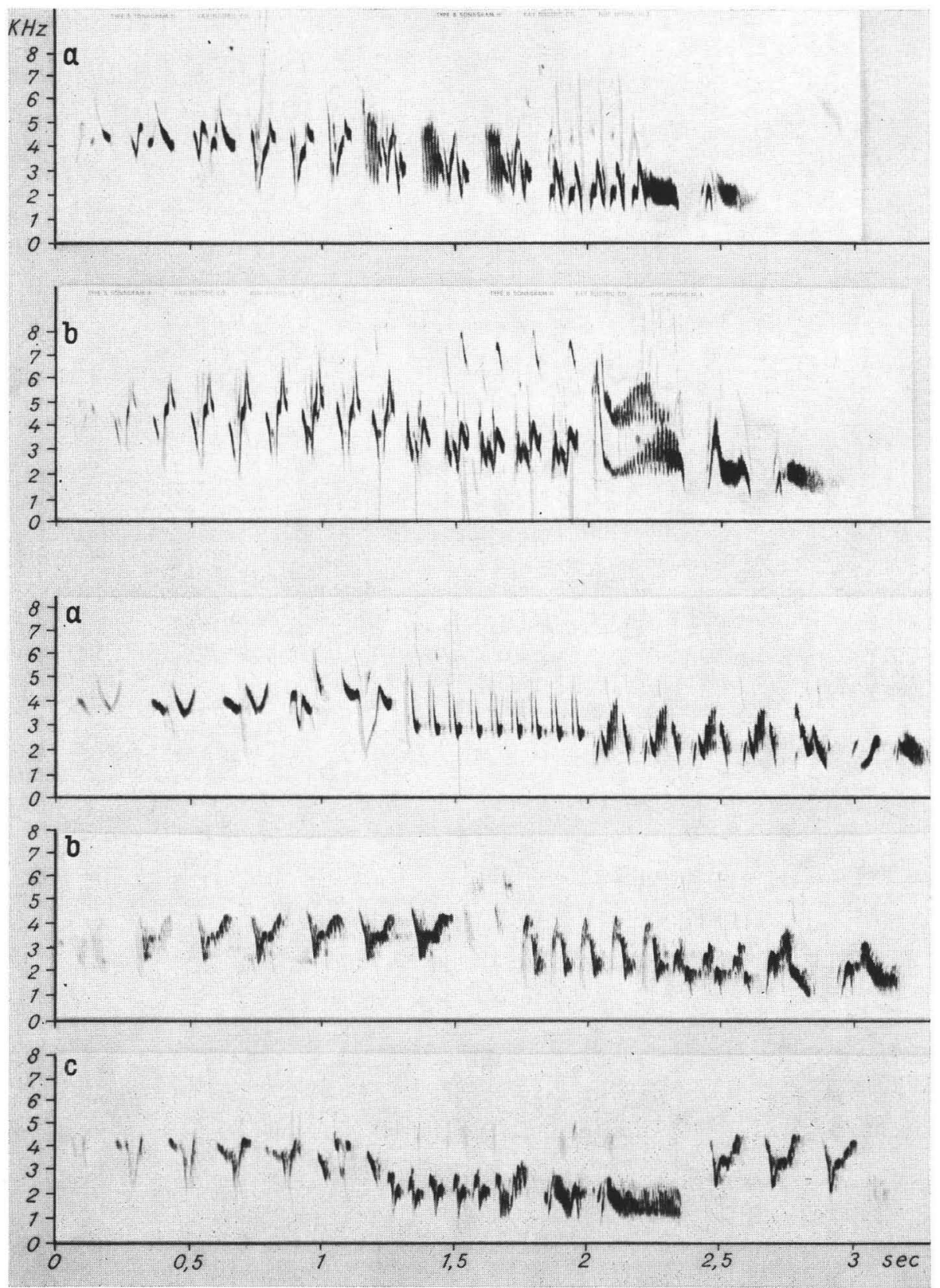

$A b b .1$ (oben) und 2 (unten): Gesangsdialekte von S. Miguel. 1. Lagoa das Furnas, 11. 5. 64. 2. Ginetes, 22. 5. 64 


\section{Motivgesang}

Der Gesang auf den Azoren ist klar zu erkennen, doch zuweilen stark vom deutschen verschieden. 74 Strophen waren im Mittel 2,5 $(1,8-3,4)$ Sek. lang. Jedes $\sigma^{7}$ besaß mindestens 2 bis höchstens 6 verschiedene Gesangsformen. Auf jeder Insel herrscht in zahlreichen, scharf begrenzten Gebieten je ein bestimmter Dialekt vor. Die Abbildungen $1-8$ zeigen solche für bestimmte Gegenden charakteristische Gesangsformen von fünf Inseln.

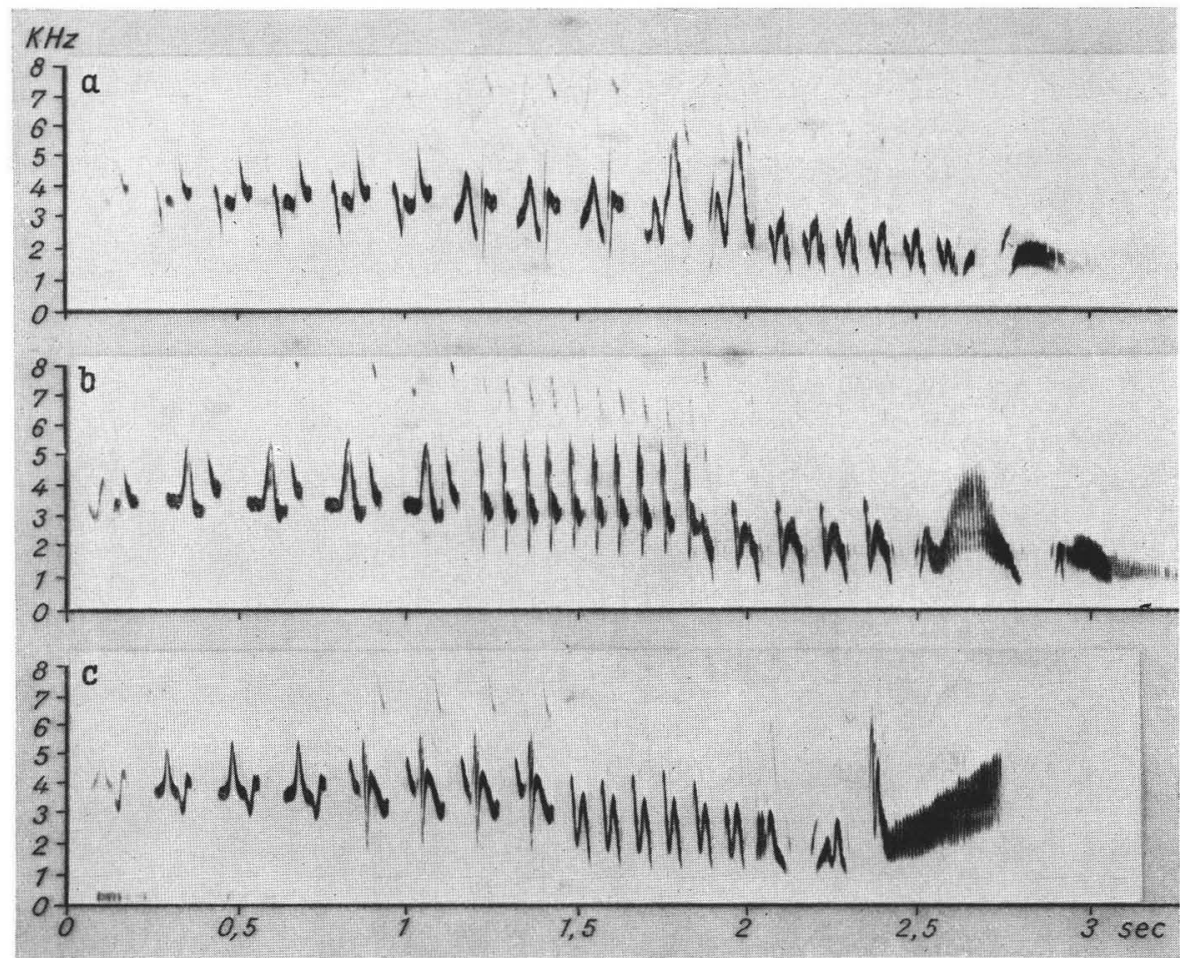

Abb. 3: Gesangsdialekt von S. Miguel. Sete Cidades, 23. 5. 64

Die drei Phrasen (Thorpe 1958) sind in einigen Beispielen gut zu erkennen (Abb. 2 a). Jeder Dialekt hat eine Gesangsform, die mit dem gleichen Endschnörkel di-djab endigt (Abb. $1-8$, jeweils a). Neben dieser häufigeren „Alltagsform" gibt es eine oder mehrere "Sonntagsformen“, deren Endschnörkel stark betont (Abb. $3 \mathrm{c}$ ) oder noch von einigen zusätzlichen Noten gefolgt sind (Abb. 2 c, 5 d, 8 b). Der Endschnörkel (= Phrase 3 b, Thorpe 1958) der kontinentalen Buchfinken schwankt im Frequenzbereich von $2-6 \mathrm{kHz}$, die "Alltagsform" der Azoren-Buchfinken aber zeigt statt eines "Schnörkels“ einen Notenkomplex von ziemlich konstanter Tonhöhe $(1,2-3,5 \mathrm{kHz})$ als Liedende, sehr ähnlich der Phrase $3 \mathrm{~b}$ einer Gruppe von Kaspar-Hauser-Vögeln von THORPE (1954 b, 1958). Es könnte sein, daß junge, noch ungeprägte Buchfinken die Azoren besiedelt haben (MARLER 1956 a). Wahrscheinlicher wurde der Endschnörkel sekundär zur einfachen Form reduziert, die der angeborenen Komponente ähnlicher ist. Das Nebeneinander von Strophen mit einfacher und ornamentaler Endphrase ist auffallend. Ursachen für die Reduktion der Endphrase sind weitgehend unbekannt - vielleicht war die Populationsdichte auf den 


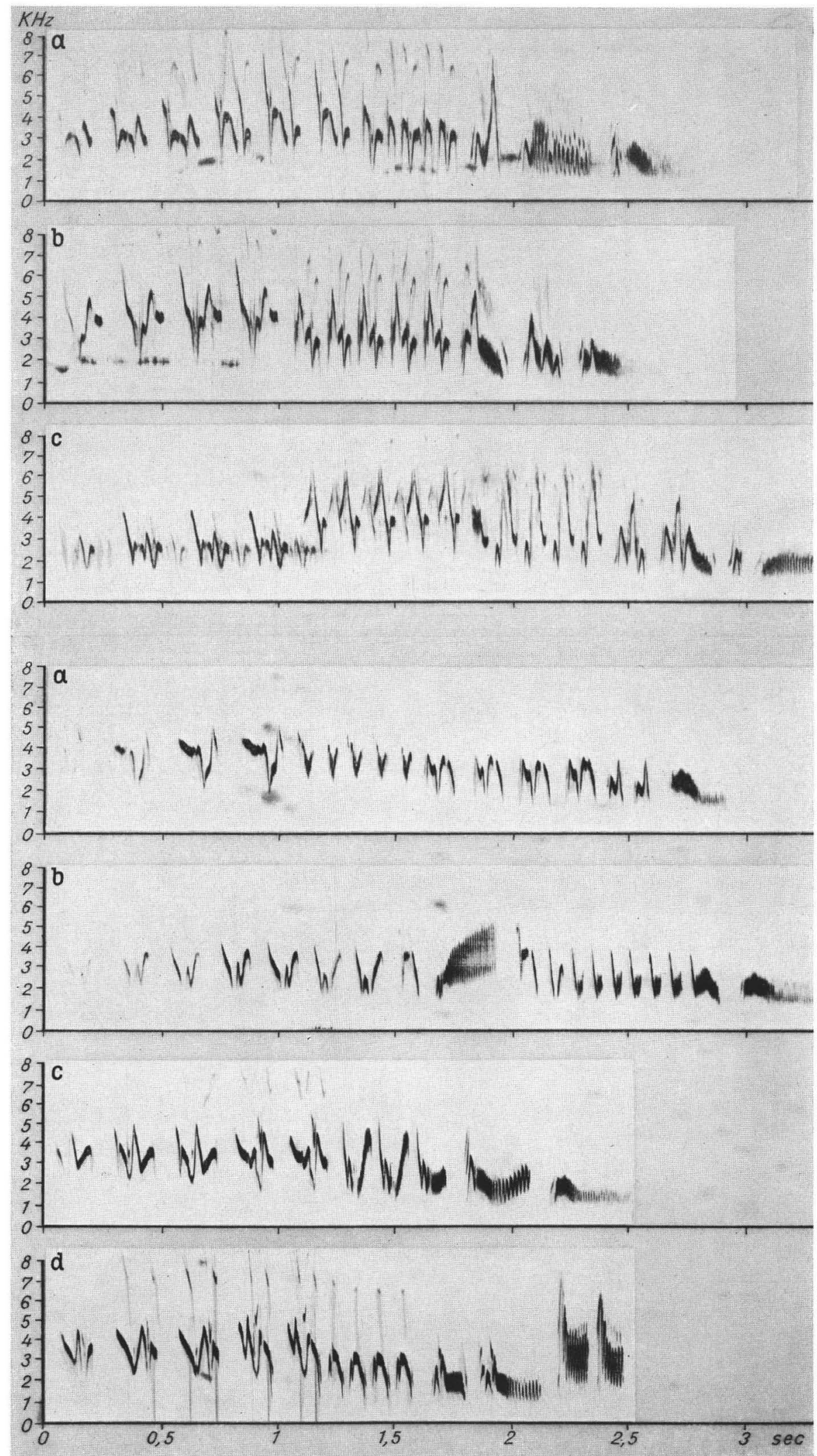

$A b b$. 4 (oben) und 5 (unten): Gesangsdialekte von S. Jorge. 4. Nähe Calheta, 24. 6. 64 5. Topo, 22. 6. 64. In der Mitte von 5b wird ein Kanarientriller imitiert (vgl. Abb. 9b) 


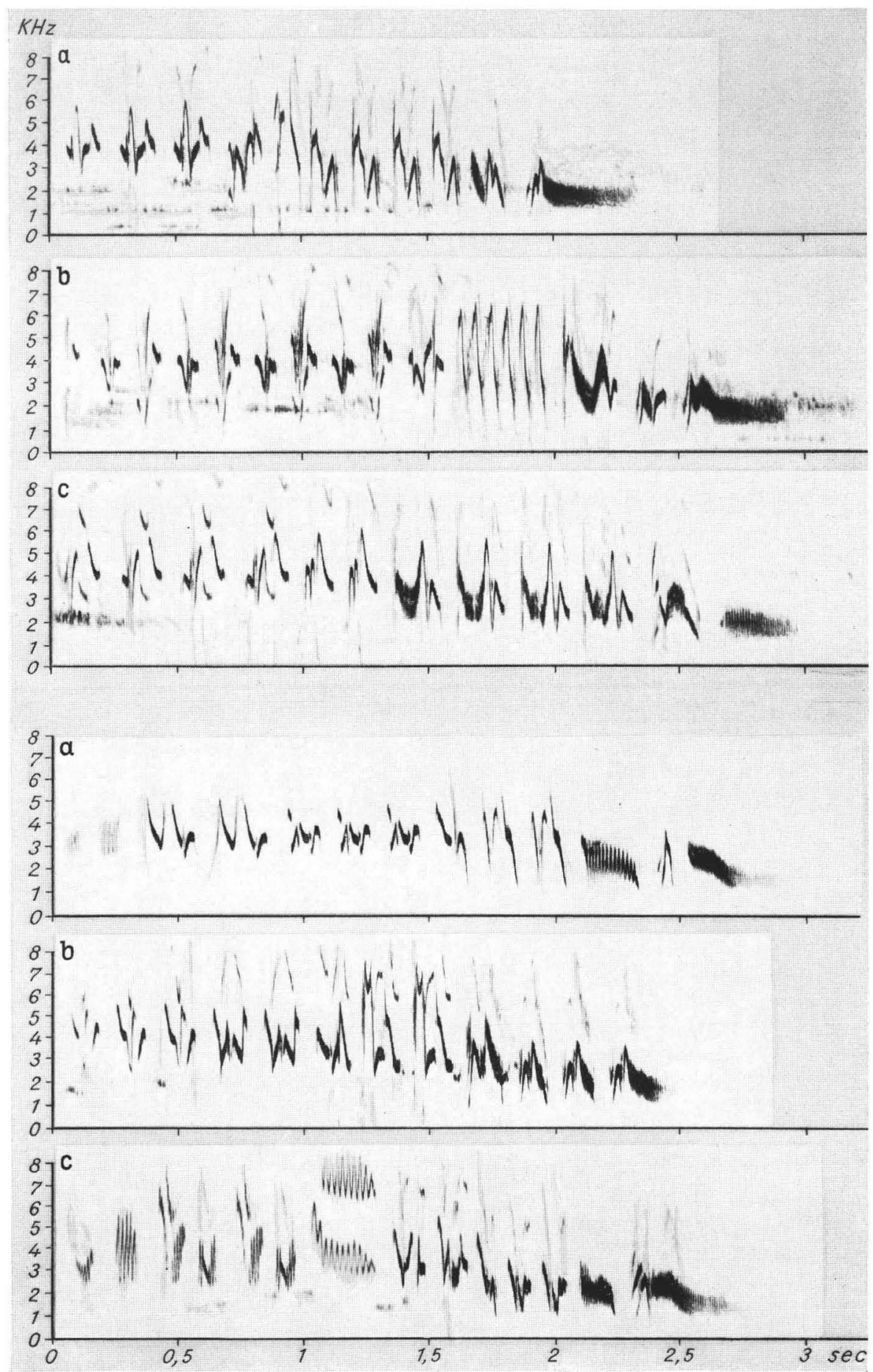

Abb. 6 (oben): Gesangsdialekt von Faial. Nähe Horta, 27. 6. 64

$A b b .7$ (unten): Gesangsdialekt von Graciosa. Feteira, 13. 6. 64. In c) wird der Gesang mit mehrfach wiederholtem Kanarientriller begonnen (vgl. Abb. 9a) 
Inseln gering und der ausgearbeitete Gesang nicht mehr so wichtig für den Bruterfolg. Die heutige Populationsdichte der Azoren-Buchfinken unterscheidet sich nicht wesentlich von der auf dem Kontinent, doch lassen sich daraus keine Schlüsse auf frühere Zeiten ziehen, da nach der Besiedlung der Inseln vom Jahr 1439 an der ursprüngliche Wald (vor allem Laurus und Erica) abgeholzt wurde und neuerdings ganz andere Bäume angepflanzt werden.

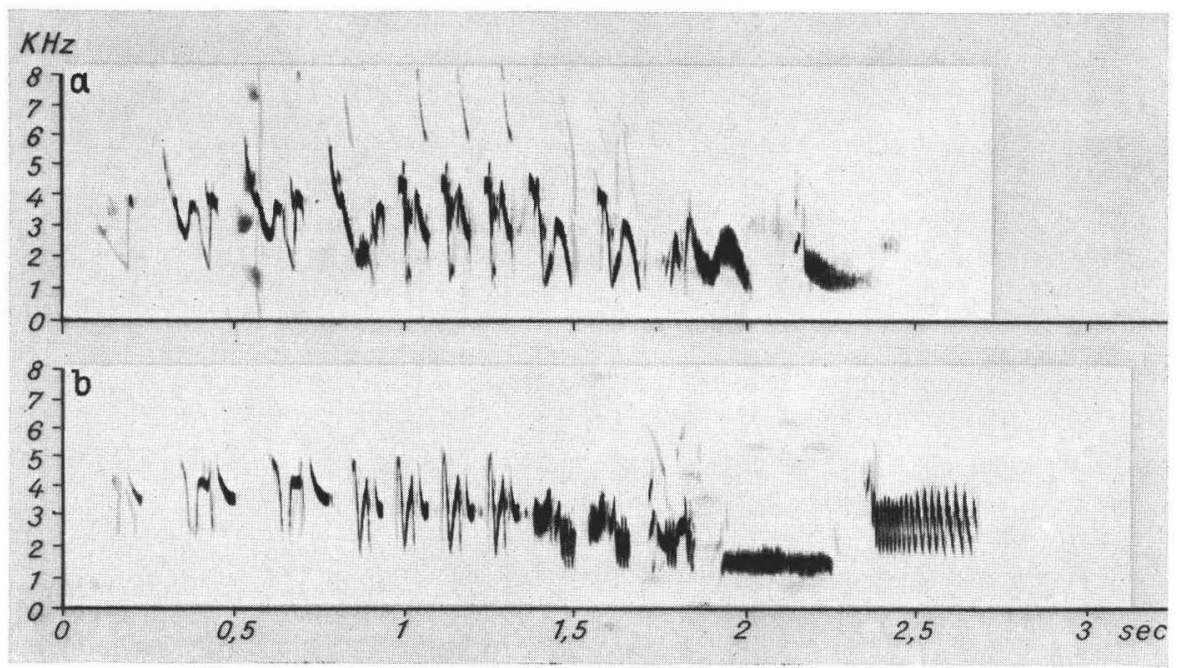

Abb. 8: Gesangsdialekt von Terceira. Mata da Serreta, 8. 6. 64

Daß die Tendenz zu einem ausgearbeiteten Endschnörkel vorhanden ist, zeigen die verschiedenen "Sonntagsformen“, die aber nie so kompliziert wie das Liedende der kontinentalen Buchfinken sind.

Phrase 1 und 2 lassen sich fast immer gut unterscheiden und erscheinen genauso variabel wie auf dem Kontinent. Doch sind öfters Triller eingefügt, die sehr an den Gesang des Kanarienvogels (Serinus canaria L.) erinnern. Schon beim Aufnehmen der betreffenden Buchfinkengesänge notierten wir, daß sie „kanarienähnlich“ geklungen hätten. Das war immer der Fall, wenn das Buchfinkenrevier im Biotop der Kanarienvögel lag (im Bereich der Kulturzone, bis zu 300 m Höhe). Die Aufnahmen von Graciosa stammen aus einem Gebiet, in dem wir doppelt soviel Kanarien- wie Buchfinkennester fanden. Der Beginn des Gesangs (Abb. 7 a , c) imitiert den Kanarientriller (Abb. 9 a). Die erste Gesangshälfte (Abb. $7 \mathrm{c}$ ) ist von einem Kanariengirlitz kaum zu unterscheiden, erst die zweite Hälfte klingt „buchfinkenähnlich“. Bei Topo auf der Insel S. Jorge kam eine für dieses Gebiet typische Gesangsform vor (Abb. 5 b) mit einem anderen leicht abgewandelten Kanarientriller (Abb. 9 b) in der Mitte.

Obwohl Poulsen (1951) und Thorpe $(1954 \mathrm{a}, 1958)$ in zahlreichen Versuchen zeigten, daß Buchfinken im Motivgesang keine anderen Vogelarten imitieren (abgesehen vom Spotten, THIELcKe 1962), also nur das „richtige“ Tonmodell beim Lernen des Gesangs benutzen, zeigen die obigen Beispiele, daß artfremde Gesangskomponenten, wenn vielleicht auch erst nach vielen Generationen, aufgenommen werden. Eine solche Abweichung vom Normaltyp des Gesangs kann sich nur dann erhalten, wenn er trotzdem die artspezifischen Funktionen erfüllt. Der Bruterfolg wird um so größer sein, je geringer die Konkurrenz und damit die Populationsdichte der Buchfinken ist. Das stimmt für die 
Kulturzone, wo die Kanarienvögel ihr Verbreitungsmaximum haben. Beides zusammen erklärt, warum das Imitieren der Kanarientriller auf diese Zone beschränkt ist.

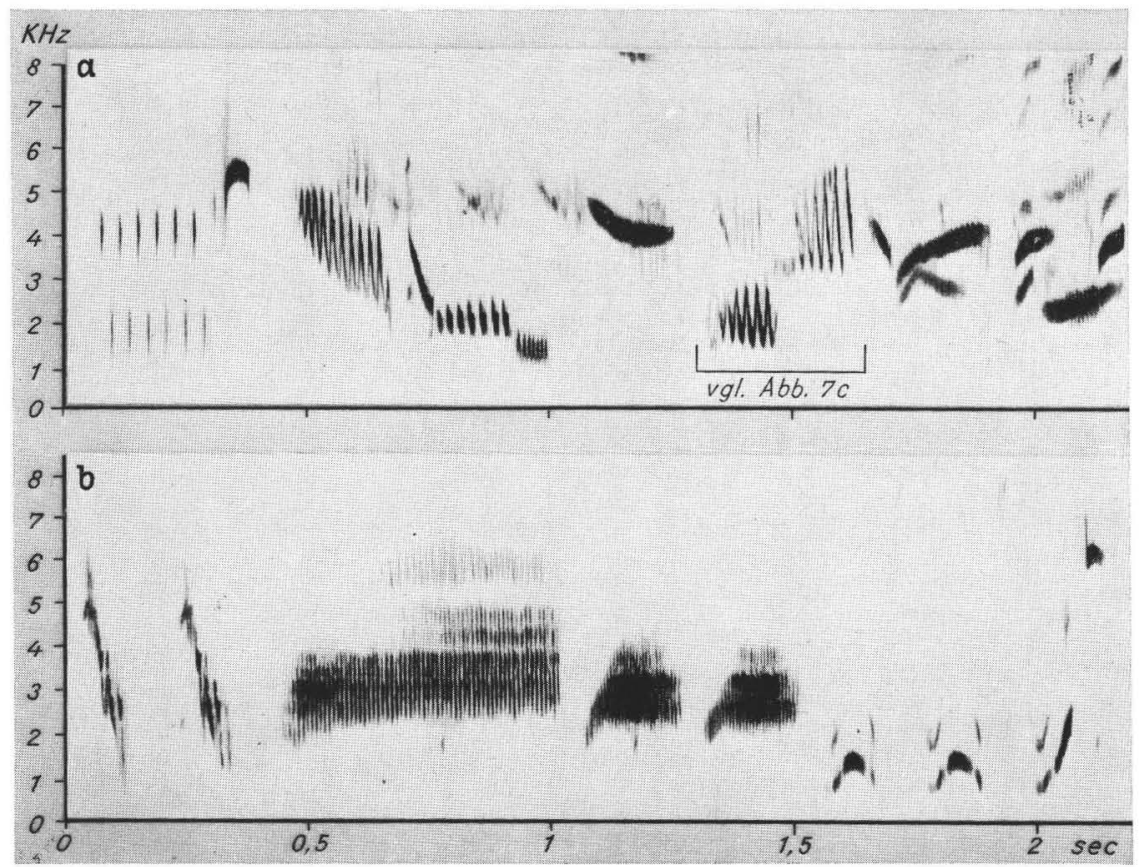

Abb. 9: Ausschnitte vom Gesang eines Kanarienvogels

Die Frage des „Rassengesangs“ könnte nur eine statistische Untersuchung beantworten. Dazu fehlen uns jedoch genügend Aufnahmen von deutschen Buchfinken. Ein statistischer Vergleich der Buchfinken von der Insel Pico mit einigen kontinentalen Dialekten findet sich bei MARLER (1952).

\section{Sozialruf (Social Call)}

Kennt man den äußeren Anlaß für eine Stimmäußerung, so wird man sie im allgemeinen auch auslösen können. Versuche dieser Art unternahmen wir im April 1964 und März-Mai 1965 in Freiburg, indem wir einem Buchfinken$O^{7}$ in seinem Revier den eigenen Gesang oder den eines Artgenossen vorspielten. Es sang zunächst heftig, näherte sich der Tonquelle und rief angriffsbereit pink-pink. Das ließ sich beliebig oft wiederholen. Wir prüften auf diese Weise mit deutschem Buchfinkengesang auch Azoren- $\sigma^{\prime} \sigma^{\prime}$. Auf allen Inseln kamen wir zu demselben Ergebnis:

a) es gibt keine „Sprachschwierigkeiten“, d. h. keine Unterschiede, ob wir einem $\sigma^{7}$ seinen eigenen oder deutschen Gesang vorspielten;

b) es kommen aber gemeinsame, wohl nur für die Azoren typische Abweichungen im ausgelösten Sozialruf gegenüber dem kontinentalen pink vor.

Versuch 1: Azoren- $\hat{o} \hat{o}$ wird der eigene oder deutscher Gesang vorgespielt. Von Mitte Mai bis Mitte Juni führten wir diesen Versuch auf verschiedenen Inseln $30 \mathrm{mal}$ durch.

Hörten wir ein singendes $\hat{\delta}$, stellten wir Tonbandgerät und Lautsprecher in seine Nähe, zogen uns zurück und beobachteten. Die Apparatur war stets ungetarnt, wir standen in 5-20 $\mathrm{m}$ Entfernung in Deckung. Doch konnten wir uns bisweilen offen bis zu $2 \mathrm{~m}$ dem Lautsprecher nähern, ohne daß der Vogel viel Notiz von uns nahm. Die Reaktion der Revierbesitzer war am Anfang stets gleich. Das $\hat{\sigma}$ schien für einige Sekunden erstarrt, streckte den 
Schnabel leicht nach oben und lauschte. Dann sprang es auf seinem Sitzplatz hin und her und besah sich den "Störenfried“ mit einem, dann mit dem anderen Auge. Konnte es den Lautsprecher anfangs nicht sehen, ortete es ihn akustisch unter mehrfachem Wechsel des Sitzplatzes. Nie wurde der Lautsprecher in gerader Linie angeflogen. Ab und zu ließ der Vogel einige ärgerliche $g \ddot{a}-g \ddot{a}$-Rufe hören.

Bei den einzelnen Versuchen wurde Verhalten und Lautäußerung von hier ab unterschiedlich. Dazu einige Tagebuchnotizen:

„31. 5. 64, Mata da Serreta, Terceira. Naturschutzgebiet, ansteigendes Gelände, dicht mit Pittosporum undulatum und Pinus maritima bepflanzt.

Reaktion des Revierbesitzers beim Abspielen seines Gesangs in Nestnähe (4 Eier): Zuhören, er umfliegt den Lautsprecher in größerem Abstand, Körnerpicken am Boden, Schnabelwetzen am Zweig, dann fliegt er geradewegs zum Lautsprecher, setzt sich neben ihn, läuft

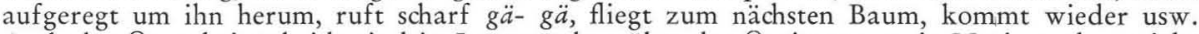
Auch das $O$ erscheint, beide sind in Lautsprechernähe, das $O$ nimmt wenig Notiz und verzieht

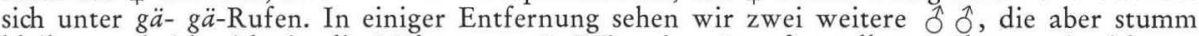
bleiben und sich nicht in die Nähe wagen." (Wie wir später feststellten, näherten sie sich nur bis zu ihrer Reviergrenze.)

„8. 6. 64, Mata da Serreta, Terceira. Das Tonbandgerät stellen wir auf einen Waldweg, der eine Reviergrenze bildet. Anfangsreaktion wie oben, dann fliegt der Vogel auf den Weg, versteckt sich in einer Entfernung von 1,5-2 m vom Lautsprecher unter Farnkräutern und beginnt dort auf dem Boden zu singen, zunächst ganz leise, dann immer lauter. Der Nachbar läßßst sich nicht blicken."

„14. 6. 64, Caldeira, Graciosa. In dem $400 \times 1000 \mathrm{~m}^{2}$ großen Einbruchkrater haben einige " $\hat{\sigma} \hat{o}$ ihre Reviere, obwohl nur wenige Büsche dort wachsen. Auf dem Kraterboden, in der Nähe der unterirdischen Grotte, hören wir $\mathrm{Nr}$. 1. Wir lassen unseren üblichen Versuch ablaufen, der Lautsprecher ist gut sichtbar. Großes Erstaunen des "Caldeirafinken", da er nur wenig Konkurrenz gewohnt ist. Unruhig fliegt er hin und her und taucht auch in die Grotte hinab, wo er den Eindringling zu finden glaubt. Nach einiger Zeit gibt er einen Ruf von sich, der etwas weicher als das gä klingt. Inzwischen haben sich zwei weitere $\hat{\jmath} \hat{\jmath}$ genähert. Zeitweilig sitzen zwei recht friedlich nebeneinander. Der dritte entfernt sich bald wieder. Nr. 2 singt in $10 \mathrm{~m}$ Abstand vom Lautsprecher und scheint weniger erregt als der Revierbesitzer Nr. 1, der sich immer wieder stumm dem Lautsprecher nähert. Doch auch er ist nicht so aufgeregt wie sonst üblich, wahrscheinlich weil er ein sehr großes Revier besitzt. $\{q$ sehen wir nicht."

„22. 6. 64, Topo, S. Jorge. Kleine Felder mit Windschutzhecken. Ganz in der Nähe eines Nests mit zwei schon älteren Jungen machen wir den Versuch. Das ồ setzt sich in einer Entfernung von $5 \mathrm{~m}$ vom Lautsprecher auf einen Ast, es hat eine Raupe im Schnabel. Trotzdem

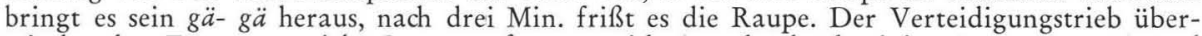
windet den Fütterungstrieb. Dann entfernt es sich (wurde durch einige Leute gestört) und beginnt zu singen, wobei der Gesang oft in Rufen endigt." nisse:

Zahlreiche Wiederholungen auf anderen Inseln brachten folgende Ergeb-

a) Beim Vorspielen innerhalb des Reviers äußert der Inhaber meist nur Rufe, versucht anzugreifen und kommt bis auf wenige $\mathrm{cm}$ an den ungetarnten Lautsprecher heran. Die gä-gä-Rufe sind mit starker Aggressionsneigung verbunden.

b) An der Reviergrenze bleibt der Besitzer in größerer Entfernung vom Lautsprecher, es kommt zu einem Singduell. Der Gesang ist mit schwacher Aggressionsneigung verbunden. Läßt sich der Nachbar blicken, können beide Vögel an der gemeinsamen Grenze kämpfen.

c) Auf den Azoren ist das in Europa übliche pink durch gä ersetzt. Beide Rufe sind in ihrer Funktion gleich; sie dienen beiden Geschlechtern als Sozialruf.

Das pink (Abb. 10 a) ist in Deutschland, England (Marler 1956 a) und Dänemark (Poulsen 1958) gleich. Es beginnt mit drei schnell aufeinanderfolgenden Noten, die einen weiten Frequenzbereich umfassen und dem Konsonan-

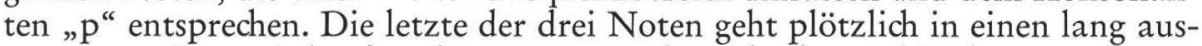
gezogenen Ton mit beschränktem Frequenzbereich, das „ink“ über.

Der Sozialruf auf den Azoren (Abb. $10 \mathrm{~b}-\mathrm{h}$ ) besteht aus mehreren simultanen Noten anstatt aus einer wie beim pink. Abb. 10 b (der Ruf läßt sich mit gi umschreiben) und das deutsche pink sind weitgehend gleich, doch neigt die 
azorische Form dazu, den Frequenzbereich durch simultane Noten zu vergrößern. Noch ausgeprägter ist das in den Spektrogrammen der anderen Inseln. Die Azoren-Buchfinken haben also einen speziellen Sozialruf, einen Rassenruf.

Er ist nicht immer aggressiv getönt, wir hörten ihn auch ohne erkennbaren äußeren Anlaß; dann klang er wesentlich weicher. Dasselbe gilt auch fürs pink (Marler 1956 a). Das weiche gä läßt sich mit djäh umschreiben, die Hauptfrequenz liegt bei $2 \mathrm{kHz}$ (Abb. $11 \mathrm{a}$ ). Mit zunehmender Erregung nehmen die

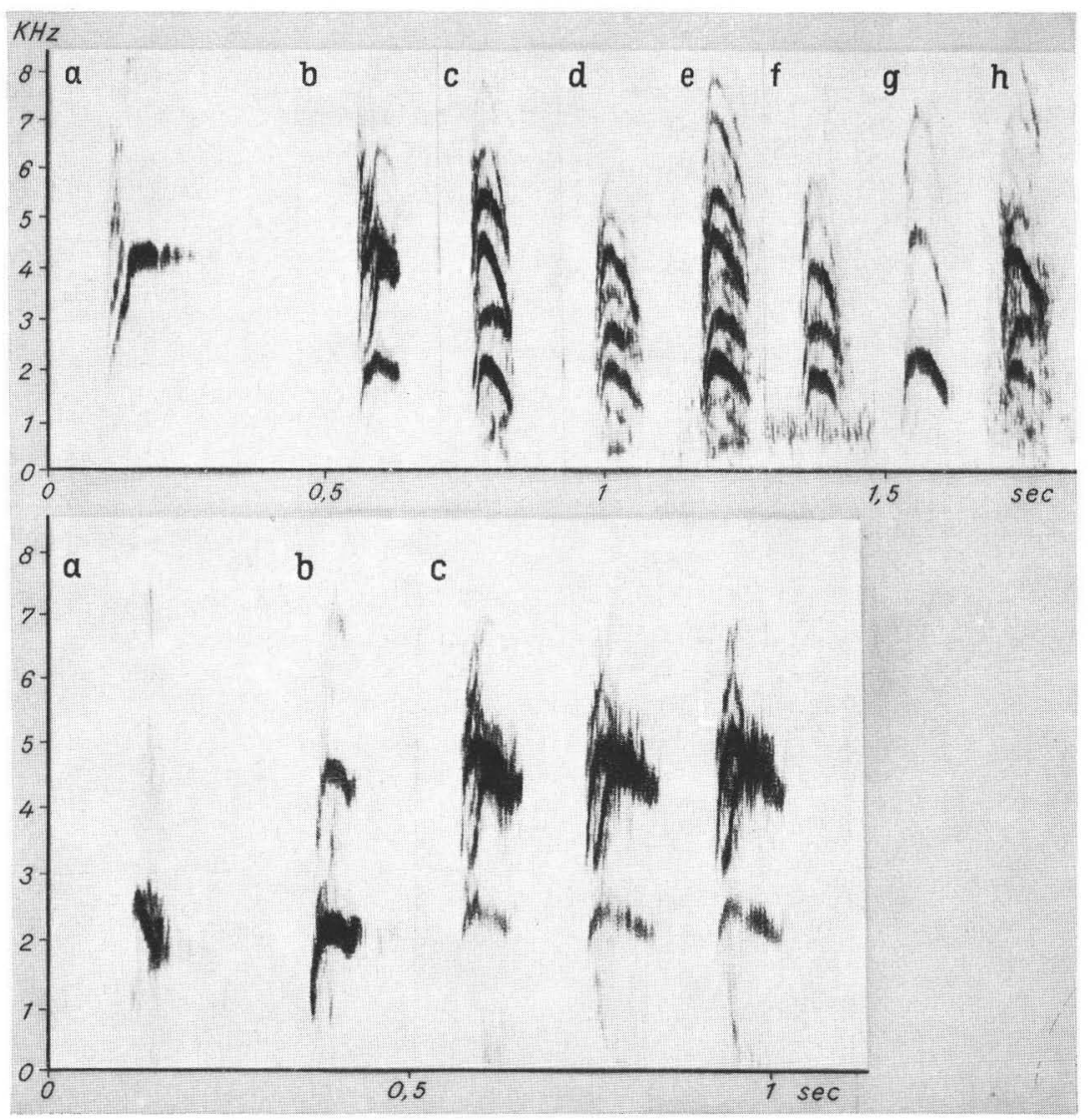

Abb. 10 (oben): Verschiedene Formen des Sozialrufs. a) kontinentale Form pink, Freiburg,

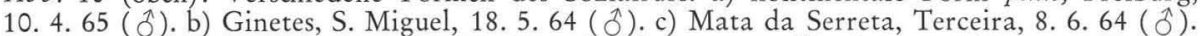
d) S. Mateus, Pico, 18.7.64 (ठ). e) Horta, Faial, 11.7.64 (q). f) Topo, S. Jorge, 21.6.64

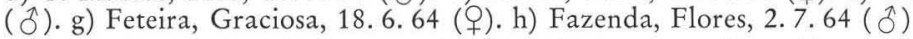

$A b b .11$ (unten): Sozialruf in verschiedenen Erregungsstadien. Ginetes, S. Miguel, 23. 5. 64 ( $($ ) $)$. a) „weiches" gä, ohne Erregung. Mit zunehmender Erregung werden die höheren Frequenzen bevorzugt (b), bis das aggressive gä erreicht ist (c)

höheren Frequenzen zu, über eine Zwischenform wird das aggressive gä erreicht $(\mathrm{Abb} .11 \mathrm{~b}, \mathrm{c})$.

Um das aggressive Verhalten und den Sozialruf auszulösen, genügten schon kurze Gesangausschnitte (etwa 1/2 Sek., mehrmals wiederholt). Auch rückwärts gespielter Gesang wurde sofort erkannt.

Selbst nach der Brutzeit konnten wir den Sozialruf auslösen. Der Gesang wird im letzten Julidrittel immer seltener, allerdings schwanken Ende der Ge- 
sangszeit und Beginn der Mauser örtlich sehr stark. Am 27.7.64 reagierten die $\sigma^{7} \sigma^{7}$ auf das Vorspielen mit Herbeifliegen und g $\ddot{a}-g \ddot{a}$-Rufen, Anfang August hatten wir mit diesem Versuch keinen Erfolg mehr. Obwohl sich die Vögel sehr wahrscheinlich noch in ihren Revieren aufhalten (weite Flüge werden während der Mauser vermieden), verteidigen sie ihre Reviergrenzen nicht mehr.

\section{Entwicklung des Sozialrufs}

Nach Marler (1956 a) entwickelt sich bei der Nominatform der Sozialruf in einem Alter von etwa fünf Wochen, gleichzeitig verschwindet allmählich der Bettelruf. Diesen hörten wir am 29.6. von einem Jungen, das bereits das Nest verlassen hatte (Abb. 12 b). Das tschirrup war deutlich mit Füttern ver-

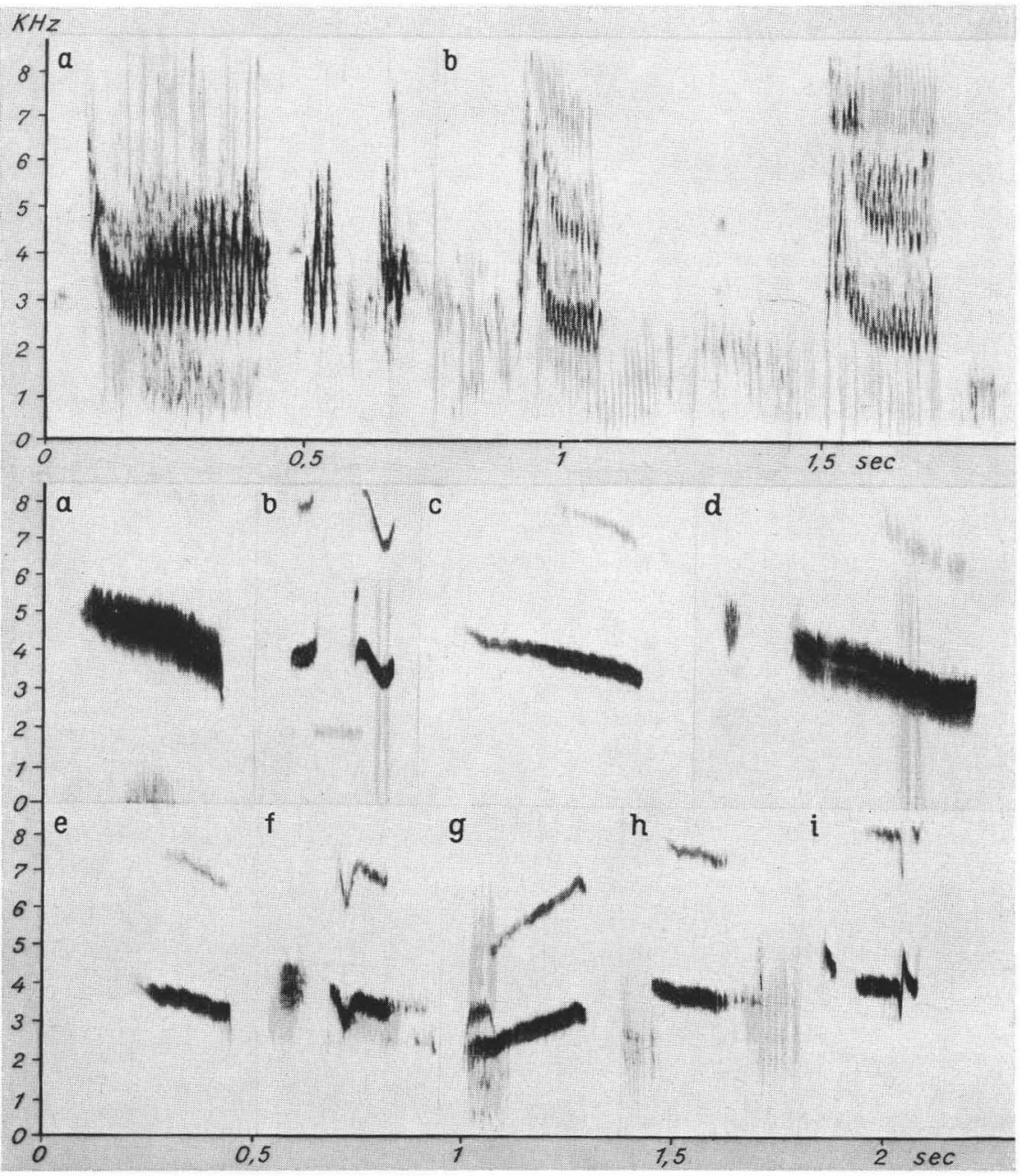

Abb. 12 (oben): Bettelrufe. a) bereits selbständiger Jungvögel, Nähe Calheta, S. Jorge, 24. 6. 64. b) mit Füttern assoziiert. Jungvogel außerhalb des Nestes. Nähe Horta, Faial, 29. 6. 64 $A b b .13$ (unten): Brutstimmungsrufe („Regenrufe“). a) dschieh, Ginetes, S. Miguel, 18. 5. 64. b) di-de, Mata da Serreta, Terceira, 8.6.64. c) siii, Nähe Calheta, S. Jorge, 24.6.64. d) di-dschieh, Topo, S. Jorge, 22.6.64. e) dii, Nähe Horta, Faial, 27.6.64. f) di-de, Matos Souto, Pico, 19.7.64. g) dubid, S. Mateus, Pico, 18.7.64. h) dii, Fazenda, Flores, 2. 7.64. i) di-di, Ribeira Fazenda, Flores, 7. 7.64 
bunden, danach verhielt sich der Jungvogel für einige Zeit ruhig. Der Ruf besteht aus Tönen, die einen weiten Frequenzbereich umfassen und schnell aufeinanderfolgen; dadurch ist der Rufer durch die Eltern sehr leicht zu orten. Sind die jungen Buchfinken unabhängig geworden, ändert der Bettelruf seine Funktion und übernimmt die Rolle des Sozialrufs. Am 24.6. sahen wir auf S. Jorge zum erstenmal kleine Trupps von 5-10 jungen, bereits unabhängigen Buchfinken, die sich gegenseitig jagten oder gemeinsam auf Futtersuche gingen. Beim Jagen ließen sie Rufe hören, die wie tschrr-da-dad klangen (Abb. 12 a). Auf vorgespielten Gesang reagierten sie nicht.

Am 7.8., wir waren inzwischen wieder auf S. Miguel, hörten wir noch immer Bettelrufe, doch antworteten nun die Jungvögel deutlich auf vorgespielten Gesang:

„Ginetes, S. Miguel, Garten mit einigen Bäumen. Beim Vorspielen fliegen aus der Umgebung acht junge Buchfinken zusammen, die sich aber in einiger Entfernung vom Lautsprecher auf den Bäumen aufhalten, wobei einige gä-gä rufen, andere tschrr-da-dad. Nur einer kommt unter lauten gä-Rufen bis auf drei $\mathrm{m}$ an den Lautsprecher heran. Nach $15 \mathrm{Min}$. versucht einer zu singen. Der Liedanfang gelingt, endigt aber im typischen Bettelruf. Nach dem $\mathrm{Ab}$ schalten zerstreuen sich die Vögel, wir hören noch einige Bettelrufe, dann sind sie still."

Von einem bestimmten Alter $\mathrm{ab}$ antworten Buchfinken auf vorgespielten Gesang („frühreifes Fortpflanzungsverhalten“, MARLER 1956 c), je nach Alter des Vogels mit dem Bettel- oder dem Sozialruf g̈̈, ein Hinweis darauf, daß beide Formen die gleiche Funktion besitzen. Die große Ähnlichkeit beider Rufe fällt auf. Der ausgebildete Sozialruf (Abb. $10 \mathrm{~b}-\mathrm{h}$ ) hat im Gegensatz zu dem der Nominatform (Abb. 10 a) einen weiten Frequenzumfang, das Kennzeichen für jugendliche Stimmäußerungen. Bei Kaspar-Hauser-Buchfinken (MARLER 1956 a) entwickelte sich der Sozialruf zuerst als ein grobes chca, das manchmal so blieb und nicht zum pink wurde. Offenbar spielt auch hier, ebenso wie bei der Ausbildung des Gesangs, Lernen eine Rolle. Der Ruf mit seiner verwischten Struktur ist dem gä der Azoren sehr ähnlich.

\section{Brutstimmungsruf (Regenruf)}

Der Ausdruck „Regenruf“ hat sich leider eingebürgert, obwohl er, wenn überhaupt, nur in den seltensten Fällen durch ein drohendes Unwetter ausgelöst wird. Wir nennen ihn den „männlichen Brutstimmungsruf“, da er nur vom $\sigma^{T} \mathrm{zu}$ hören und an die Fortpflanzungszeit gebunden ist. LEPIKSAAR (1942) nennt ihn „Brutzeitruf“. Über die geographische Variation dieses Rufs unterrichten Promptoff (1930), Sick (1939, 1950), Lepiksaar (1942), Ringleben (1942), Schulz (1942), Stresemann (1942, 1943), Lunau (1943), Krätzig (1943), Wolters (1943), Poulsen (1958). Eine Zusammenfassung der verschiedenen Rufformen geben StrREsEMANN (1943) und Sick (1950). Danach sind die einzelnen Formen mosaikartig verteilt. Nach Sick (1950), Peitzmeier (1955) und Sokolowski (1965) werden die Rufe bei mäßiger Gefahr, aber auch spontan geäußert. Bei akuter Gefahr werden pink-Rufe eingefügt.

Wie auf dem Kontinent, ist auch der Brutstimmungsruf der Azoren-Buchfinken stark variabel (Abb. 13); die einzelnen Formen können innerhalb kleiner Gebiete sprunghaft wechseln, oft ohne erkennbare geographische Barrieren. Am häufigsten hörten wir Rufe wie dschieb (Abb. 13 a) oder diii (Abb. 13 c, e, h), auf Pico nahmen wir einen auf, der dem huit-Alarmruf des Kontinents sehr ähnlich ist (Abb. $13 \mathrm{~g}$ ). Alle Rufformen kommen möglicherweise auch bei europäischen Buchfinken vor, sie sind wohl nicht spezifisch für die Azoren.

Der Vogel saß oft ruhig auf einem Ast und wiederholte den Ruf ohne erkennbaren äußeren Anlaß monoton 30-70mal pro Minute. Auch bei mäßiger Gefahi - wir hatten den Parabolspiegel in einem Revier aufgebaut - wurde 
der Brutstimmungsruf ausgelöst, dann allerdings mit gä-Rufen vermischt. Bei akuter Gefahr, vermaßen wir z. B. ein Nest, umflogen uns die Eltern aufgeregt und ließen ihren aggressiven Sozialruf hören. Entfernten wir uns, so beruhigten sie sich schnell, das $\sigma^{7}$ gab den Brutstimmungsruf von sich, das $q$ ein weich klingendes djäb (Abb. 11 a).

Den kontinentalen buit-Alarmruf (nicht zu verwechseln mit dem buidBrutstimmungsruf) hörten wir nie. Die Azoren-Buchfinken haben als stärksten Warnruf das aggressive gä (und nicht buit), als gemilderten Laut den Brutstimmungsruf (vgl. SicK 1939).

\section{Flugruf}

Den Flugruf konnten wir nur einmal auf S. Jorge am 21. 6. von einem $\sigma^{7}$ aufnehmen (Abb. 14). Der Vogel ließ das tjup kurz vor dem Auffliegen hören. Der Ruf scheint mit dem vom Kontinent übereinzustimmen, doch fehlen uns weitere Aufnahmen.

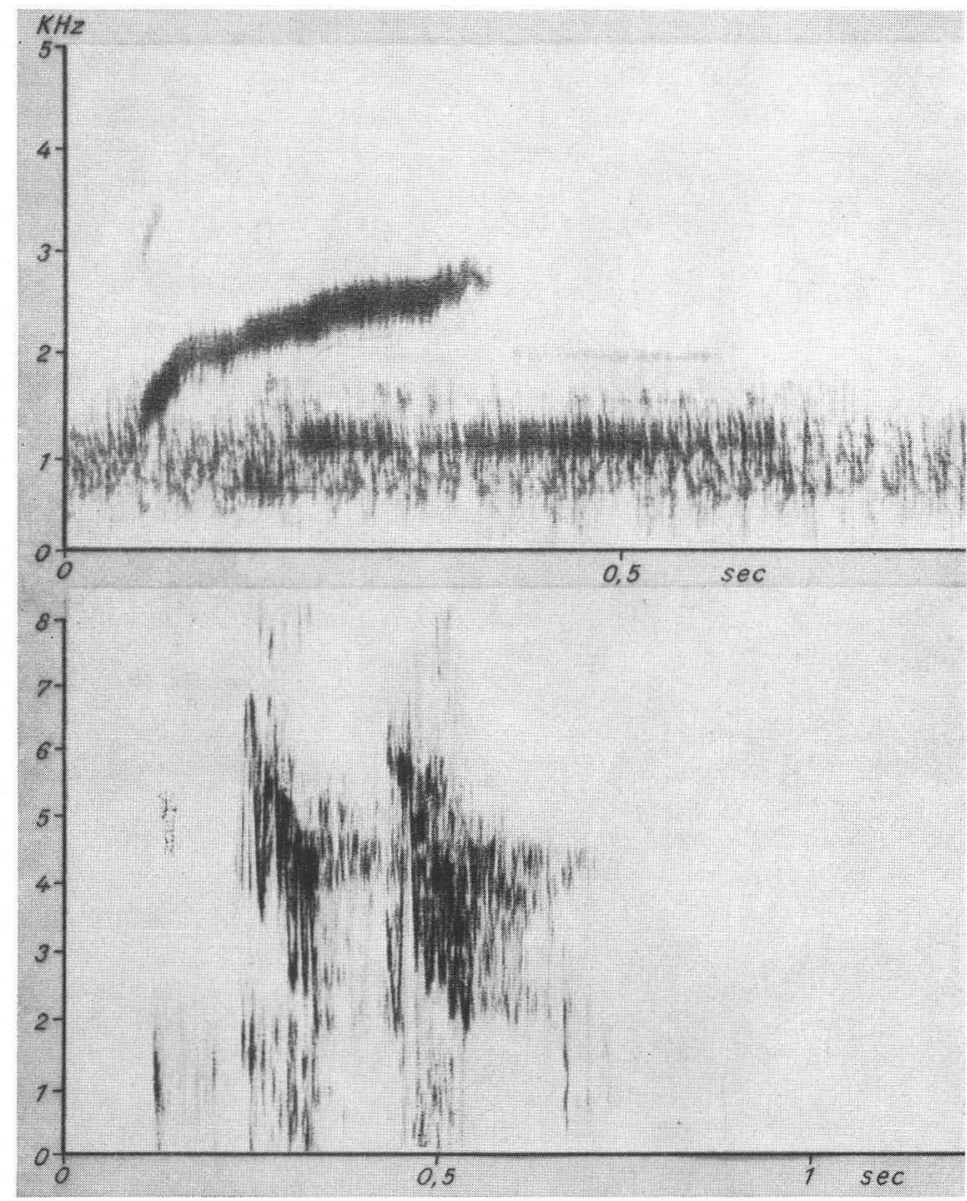

Abb. 14 (oben): Flugruf tiup eines ô. Topo, S. Jorge, 21. 6. 64

$A b b .15$ (unten): Alarmruf tschrid-tschrid eines noch nicht flüggen Jungvogels außerhalb des Nestes. Ginetes, S. Miguel, 26. 5. 64 


\section{Alarmruf eines Jungvogels}

Am 19. 7. fanden wir auf Pico in einem Areal von $50 \mathrm{~m} \times 25 \mathrm{~m}$, das dicht mit $15 \mathrm{~m}$ hohen Akazien bestanden war, 8 Buchfinkennester. In einem saßen zwei schon ältere Junge, die anderen waren leer. Auf dem Boden hüpfte ein noch nicht ganz flügger Buchfink. Als wir ihn fingen, ließ er einige Alarmrufe hören (Abb. 15), die den Jungenrufen (Abb. 12) sehr ähnlich sind. Innerhalb von Sekunden versammelten sich darauf mindestens 10 Buchfinken beiderlei Geschlechts unter lautem gä-g̈̈ rings um uns. Das lockte noch mehr Altvögel an. Die Anziehungskraft des Sozialrufs auf Artgenossen hatten wir schon früher festgestellt.

\section{Bestimmung der Reviergröße}

Die Größe eines Reviers läßt sich recht genau bestimmen, indem man den Besitzer an einer "akustischen Leine“ hinter sich herzieht. Öber eine bestimmte Grenze, an der er oft Kämpfe mit seinen Nachbarn zu bestehen hat, wagt er sich nicht hinaus.

Bei einigen Wiederholungen des Versuchs war das Ergebnis stets gleich, es genügt daher eine Beschreibung (Abb. 16):

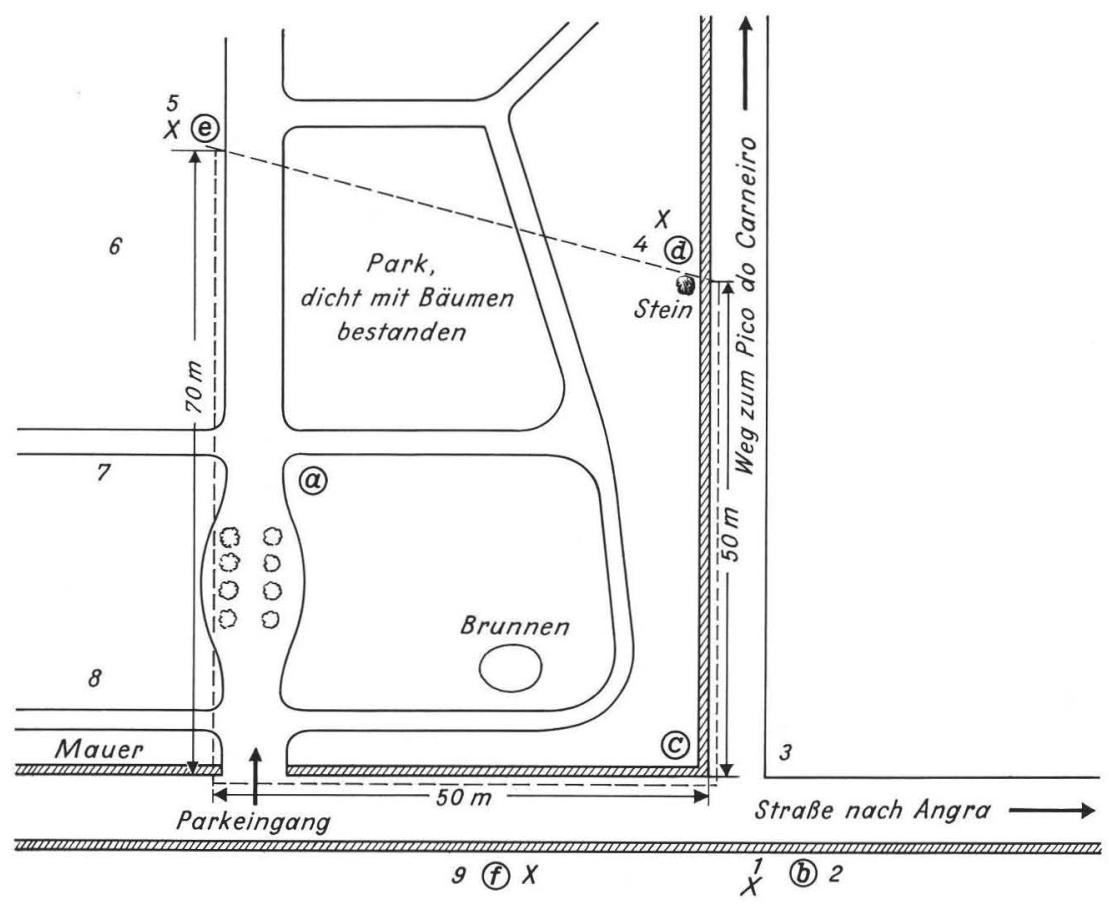

Abb. 16: Skizze eines Buchfinkenrevieres. Mata da Serreta, Terceira, 7. 6. 64. X $=$ Kampf mit Nachbar

„7. 6. 64, Mata da Serreta, Terceira. Dem Revierbesitzer "Q“ spielen wir in immer größerer Entfernung vom Nest (a) seinen Gesang vor. Langsam folgt er uns in 3-5 m Höhe, fliegt von Ast zu Ast und läßt sein aggressives gä-gä hören. Bei b erscheint Nachbar 1 und ruft gä, Q ist nun stumm. Beide kämpfen dreimal kurz, zuvor deutet sich der Kampf durch Hängenlassen und Zittern der Flügel an. Weiter nach rechts folgt uns $Q$ nicht mehr, er fliegt nach $\mathrm{c}$ und beginnt dort zu singen. Die Nachbarn 2 und 3 erscheinen, 2 bleibt stumm, 3 fängt zunächst ganz schüchtern mit seinem Gesang an, allmählich wird er lauter. Die Straße zum Pico do Carneiro hinauf folgt uns Q $50 \mathrm{~m}$ weit. Über die Mauer weiter nach rechts wagt er sich nicht hinaus. Bei d erscheint Nachbar 4 aus dem Park, beide kämpfen. Bei e muß Q mit Nachbar 5 kämpfen. Die Nachbarn 6,7 und 8 erscheinen an den eingezeichneten Stellen, es kommt allerdings zu keinem Kampf. Q wagt sich nicht nach links über den Parkweg, er singt 
an seiner Grenze, die wir entlang des Weges annehmen. Bei einem Kontrollversuch etwas später kämpft $Q$ auch noch bei f mit Nachbar 9, so daß auch hier die Grenze sicher ist. Q beherrscht eine Fläche von $50 \mathrm{~m} \times 60 \mathrm{~m}$."

Die Reviergrenzen liefen oft parallel zu Mauern, Wegen und Hecken, sie waren deutlich markiert. Die Straße bildete ein „Niemandsland“, Q wagte sich allerdings hinüber, wurde dann aber von seinen Nachbarn entsprechend aggressiv empfangen. Er schien genau zu wissen, daß er in fremdes Gebiet eindrang, denn er war viel weniger angriffslustig als seine Nachbarn; stets gab Q im fremden Revier den Kampf auf und flog weg. Am besten zeigte sich seine Konfliktsituation bei Abwesenheit des Rivalen; dann wechselte er dauernd zwischen Lautsprecher und seiner Reviergrenze. Erst an seiner Grenze wagte er zu singen, außerhalb des Reviers blieb er stets stumm.

\section{Zusammenfassung}

Es wird untersucht, ob die Azoren-Buchfinken „Rassengesang “ und „Rassenrufe" haben. Gesänge und Rufe wurden auf Tonband aufgenommen und klangspektrographiert.

Motivgesang. Jedes $\sigma^{7}$ beherrscht $2-6$ verschiedene Gesangsformen, wobei stets eine "Alltagsform" mit der stark vereinfachten Phrase di-djab endigt. Die anderen, weniger häufigeren Gesangsformen ("Sonntagsformen“) zeigen eine besser ausgearbeitete Endphrase, die jedoch nie so kompliziert wie bei kontinentalen Buchfinken ist.

In Gebieten, in denen sich bevorzugt Kanarienvögel aufhalten, können Buchfinken Gesangselemente übernehmen.

Sozialruf. Das kontinentale pink ist auf allen Azoreninseln durch gä ersetzt, so daß man von einem Rassenruf sprechen kann. Er ist mit starker Aggressionsneigung verknüpft. Der Sozialruf zeigt einen weiten Frequenzumfang, hervorgerufen durch mehrere simultane Noten.

Brutstimmungsruf (Regenruf). Eine Anzahl verschiedener Rufe wurde spektrographiert. Vom $\sigma^{7}$ ist er bei mäßiger Gefahr, aber auch spontan (30-70 Rufe/Min.) zu hören.

Flugruf. Er scheint mit dem Flugruf der Nominatform identisch zu sein.

Bestimmung der Reviergröße. Ein $\sigma^{7}$ wurde innerhalb seines Reviers an die "akustische Leine“ genommen und bis zu den Reviergrenzen gezogen. Verhalten und Lautäußerung änderten sich in Abhängigkeit von der jeweiligen Entfernung bis zur Reviergrenze.

\section{Summary}

The attempt was made to determine whether Azores chaffinches possess a "racial song" or "racial calls". The songs and calls were tape-recorded and sound-spectrographs were prepared.

1. The song motif. Each $\sigma^{7}$ possesses 2-6 different song types, among which there is an "everyday type" which always ends with the greatly simplified phrase: dee-chab. The other, less frequent song types ("Sunday types") exhibit a more developed final phrase, though this is not as complex as that of Continental chaffinches.

In areas where canaries commonly occur, chaffinches may adopt some of their song elements.

2. The social call. The Continental pink is replaced by gai in all of the Azores island forms, so it is justifiable to speak of a "racial call". This call is correlated with a strong aggressive tendency; it exhibits a broad frequency range based upon simultaneous utterance of several notes.

3. The brooding call ("rain-call"). A number of different calls were spectrographed. With the $\sigma^{7}$, this call can be heard in response to mild danger and also as a spontaneous utterance (30-70 calls/min.). 


\section{type. \\ 4. Flight call. This seems to be identical to the flight call of the nominate}

5. Determination of territory size. A $O^{7}$ was led within his territory on an "acoustical lead" and drawn to his territorial boundaries. His behaviour and vocalizations altered in relation to the distance from the territorial boundary.

\section{Literaturverzeichnis}

Ausobsкy, A. (1964): Tonbandjagd auf Tierstimmen, Kosmos, Stuttgart - Frey, R. (1938): Iter entomologicum et botanicum ad insulas Madeiram et Azores. Soc. Sci. Faun. Flor. Fennica, Helsinki - Knecht, S. (1961): Ein Beitrag zur Kenntnis der azorischen Vogelwelt. Anz. Orn. Ges. Bayern 6, 121-137 - Krätzig, H. (1943): Der Regenruf des Buchfinken auf der Krim. Orn. Mber. 51, 101 - Lepiksaar, J. (1942): Über den „Regenruf“ des männlichen Buchfinken in Estland. Orn. Mber. 50, 127-128 - Lunau, C. (1943): Der Regenruf des Buchfinken in Schleswig-Holstein. Orn. Mber. 51, 140-141 - Marler, P. (1952): Variation in the song of the Chaffinch Fringilla coelebs. Ibis 94, 458-472 - Ders. (1956 a): The voice of the Chaffinch and its functions as a language. Ibis 98, 231-261 - Ders. (1956 b): Über die Eigenschaften einiger tierlicher Rufe. J. Orn. 97, 220-227 - Ders. (1956 c): Behaviour of the Chaffinch Fringilla coelebs. Behaviour Suppl. 5 - Ders. (1957): Specific distinctiveness in the communication signals of birds. Behaviour 11, 13-39 - Ders. u. D. J. Boatman (1951): Observations on the birds of Pico, Azores. Ibis 93, 90-99 - Niethammer, G. (1937): Handbuch der Deutschen Vogelkunde. Bd 1. Akademische Verlagsanstalt, Leipzig - Peitzmeier, J. (1955): Zur Deutung des „Regenrufes“ der Buchfinken. J. Orn. 96, 147-152 • Poulsen, F. (1951): Inheritance and learning in the song of the Chaffinch Fringilla coelebs L. Behaviour 3, 216-228 - Ders. (1958): The calls of the Chaffinch (Fringilla coelebs L.) in Denmark. Dansk Ornith. Foren. Tidsskr. 52, 89-105 - Promptoff, A. (1930): Die geographische Variabilität des Buchfinkenschlages (Fringilla coelebs L.). Biol. Zbl. 50, 478-503 - RINGLEBEN, H. (1942): Der Regenruf des Buchfinken in Rußland. Orn. Mber. 50, 174-175 • Schulz, G. E. F. (1942): Zum Regenruf des Buchfinken. Orn. Mber. 50, 88 - Sick, H. (1939): Ưber die Dialektbildung beim Regenruf des Buchfinken. J. Orn. 87, 568-592 - Ders. (1950): Der Regenruf des Buchfinken (Fringilla coelebs). Vogelwarte 15, 236-237 - Sokolowski, J. (1965): Wann läßt der Buchfink den „Regenruf“ hören? Ardea 53, 73-78 - Stresemann, E. (1942): Der Regenruf des Buchfinken und sein geographisches Variieren. Orn. Mber. 50, 60-61 - Ders. (1943): Über das geographische Abändern des Regenrufes von Fringilla coelebs. Orn. Mber. 51, 139-140 - Thielcke, G. (1962): Die geographische Variation eines erlernten Elements im Gesang des Buchfinken. Vogelwarte 21, 199-202 • THORPE, W. H. (1954 a): The process of song learning in the Chaffinch, as studied by means of the sound spectrograph. Nature 173, 465-467 - Ders. (1954 b): The analysis of bird song with special reference to the song of the Chaffinch. Acta 11. Congr. Int. Orn., 209-217 - Ders. (1958): The learning of song patterns by birds, with special reference to the song of the Chaffinch Fringilla coelebs. Ibis 100, 535-570 - Wahlström, S. (1960): Tonbandjagd auf Vogelstimmen. Funkschau 19, 483-486 - Wolters, H. E. (1943): Über den Regenruf des Buchfinken. Orn. Mber. 51, 98 - 99.

Anschrift der Verfasser: 78 Freiburg i/Br., Runzestraße 81. 